\title{
ESCORE DE HEMOSSIDERINA EM CITOLOGIA DE LAVADOS TRAQUEAIS PARA AVALIAÇÃO DA HEMORRAGIA PULMONAR INDUZIDA POR EXERCÍCIO EM CAVALOS DE PÓLO
}

\author{
Katia Moreira Silva ${ }^{1}$, Juliana Nabuco Pereira Otaka², Maria Luisa Abreu Loredo \\ Jorge $^{3}$, Carlos Alexandre Paula Gonçalves ${ }^{1}$, Nayro Xaxier Alencar ${ }^{1}$, Daniel Augusto \\ Barroso Lessa ${ }^{1}$ \\ 1 UFF \\ 2 Jockey Club Brasileiro \\ 3 Polícia Militar RJ \\ Correspondência: Katia Moreira Silva: katitinhabr@yahoo.com.br
}

RESUMO: O pólo é um dos mais antigos esportes equestres, tendo sido introduzido no Brasil na década de 20 e sua atividade vem crescendo desde então. $O$ aparelho respiratório é fundamental para a saúde e bom desempenho atlético dos equinos, sendo os processos mórbidos neste sistema responsáveis por prejuízos orgânicos e econômicos consideráveis. Dentre as enfermidades de maior importância do trato respiratório equino, destaca-se a Hemorragia Pulmonar Induzida Pelo Exercício (HPIE). A citologia de lavado traqueal é considerada mais específica do que somente o exame endoscópico no diagnóstico dessa enfermidade. Considerando que os equinos de pólo ainda são pouco explorados no que se refere a estudos clínicos, este trabalho teve por objetivo aplicar o escore total de hemossiderina (ETH) na citologia traqueal de animais de pólo. Foram utilizados 37 equinos participantes de atividades de pólo, que foram avaliados 30 a 90 minutos após a participação em partida. Os animais foram divididos em 2 grupos considerando a presença de sangue no exame endoscópico pós exercício. Considerando o valor de escore proposto por Doucet e Viel (2002), no grupo 1, a ocorrência de HPIE foi observada em $11,5 \%$ dos animais e no grupo 2 em $27,2 \%$. Apesar de estarem aparentemente assintomáticos, os animais apresentaram quadros citológicos compatíveis com HPIE em proporções relevantes, fato que deve levar essas enfermidades a serem consideradas como uma das primeiras opções de diagnóstico na investigação de queda de desempenho atlético de equinos nessa atividade esportiva. Em virtude de o ETH ter sido desenvolvido para o LBA, novos estudos devem ser realizados para que seja estabelecido o ponto de corte ideal para o ETH em lavado traqueal.

Palavras-chave: cavalos de pólo; hemossiderina; lavado traqueal; endoscopia; HPIE

\section{TRACHEAL WASH HEMOSSIDERIN SCORE IN EXERCISE INDUCED PULMONARY HEMORRHAGE IN POLO HORSES}

\begin{abstract}
Polo is one of the most ancient equestrian sports. It was introduced in Brazil in the twenties and has increased since then. The respiratory tract is essential for health and athletic performance in horses, and respiratory disease cause considerable organic and economic losses. Exercise Induced Pulmonary Hemorrhage is within the most important respiratory diseases. Tracheal wash cytology is more specific than only endoscopy in the diagnosis of this disease. Considering that very few clinical studies have been conducted on polo horses, the aim of this paper is to apply the total hemosiderin score (THS) in tracheal wash cytology of polo horses. A total of 37 polo horses were evaluated 30 to 90 minutes after the game. The animals were divided in 2 groups considering the presence of blood on endoscopic examination post-exercise. According to score values proposed by Doucet and Viel (2002), EIPH occurred in 11.5 and $27.2 \%$ of animals in group 1 and 2, respectively. Although apparently asymptomatic, the horses showed cytology profile compatible with $\mathrm{EIPH}$ in relevant proportions, which confirms this disease as an important cause in the diagnosis of poor performance. Since the THS was developed for BAL, new studies must be done to determine the THS cutoff in tracheal wash.
\end{abstract}

Key Words: polo horses; hemosiderin; tracheal wash; endoscopy; EIPH 


\section{INTRODUÇÃO}

O estudo das secreções obtidas do trato respiratório já vem sendo utilizado no Brasil e tem sido considerado de grande valia, já que representa um meio semiológico importante, ajudando no diagnostico das doenças ( Michelotto et al. 2007 ).

A citologia de lavado traqueal (LT) é considerada mais específica do que somente o exame endoscópico, sendo descrita como uma ferramenta no diagnóstico da doença pulmonar (Dixon, 1995).

Dentre as enfermidades respiratórias de equinos de esporte, destaca-se a hemorragia pulmonar induzida por exercício (HPIE) pela sua alta frequência, seus danos a saúde do animal e prejuízo econômico com perdas de dias de treinamento. 0 exame endoscópico da traquéia após o exercício é o método que tem sido amplamente utilizado para diagnóstico da HPIE. Porém, em alguns casos nos quais o diagnóstico endoscópico é negativo, a citologia torna-se fundamental devido à observação de células características dos processos hemorrágicos, tais como os hemossiderófagos (Doucet e Viel, 2002; Hodgson e Hodgson, 2003; Biava et al. 2008) .

Apesar do lavado broncoalveolar (LBA) ser o método de eleição para colheita de material para a avaliação citológica do trato respiratório inferior, Hoffman (2008) recomenda que 0 animal permaneça em repouso nas 24 a 48 horas posteriores à colheita. No caso de animais atletas em atividade constante, como os utilizados no pólo, esse repouso dificulta a realização do procedimento. Nessas situações, o LT pode ser utilizado, uma vez que não há necessidade de repouso após a sua realização.

A presença dos hemossiderófagos em secreções traqueobrônquicas tem sido estudada há algum tempo, porém, poucas tentativas têm sido realizadas a fim de quantificar esta observação (Doucet e Viel, 2002). Alguns autores afirmam que praticamente a totalidade dos cavalos em treinamento tem hemossiderófagos em lavados traqueobrônquicos (Whitwell e Greet, 1984). Sweeney et al. (1992) realizaram um estudo em animais de corrida aparentemente saudáveis utilizando para a colheita do aspirado traqueal a técnica via trans-traqueal e observaram hemossiderófagos em $86 \%$ destes animais. McKane et al. (1993) por meio da realização de lavado broncaolveolar hemossiderófagos em $90 \%$ das amostras.

O escore total de hemossiderina (ETH) tem sido descrito e utilizado em citologia de lavados broncoalveolares para avaliar a gravidade dos episódios de hemoptise em humanos (GOLDE et al., 1975) e HPIE em equinos (DOUCET e VIEL, 2002). Utilizando esse escore, Biava et al. (2008) encontraram $58 \%$ de cavalos da raça Quarto-de-milha com HPIE, mesmo sem evidências endoscópicas de sangramento. Quanto a utilização desse escore em lavados traqueais, DeHeer e Mc Manus (2005) o aplicaram para avaliação de felinos com doenças respiratórias.

Nesse contexto, o objetivo deste trabalho foi aplicar o escore total de hemossiderina $(\mathrm{ETH})$ na citologia de lavados traqueais para avaliação da hemorragia pulmonar induzida por exercício em cavalos de pólo após o exercício.

\section{MATERIAL E MÉTODOS}

Foram utilizados 37 equinos adultos, mestiços de Crioulo com Puro Sangue Inglês, com idade entre três a 22 anos (em média 7 anos) sendo 21 fêmeas e 16 machos, regularmente utilizados em jogos de pólo na cidade do 

hemorragia pulmonar induzida por exercício em cavalos de pólo

Rio de Janeiro. Os animais foram mantidos em cocheiras com água ad libitum, alimentados com ração comercial, aveia, feno de alfafa e suplementados com sal mineral. Eram periodicamente vermifugados e vacinados contra tétano, influenza, encefalomielite leste, oeste e raiva.

Todos os animais apresentaram frequência cardíaca, respiratória e temperatura corpórea dentro dos valores de normalidade segundo $\mathrm{Mc}$ Gorum et al. (2002), assim como resultados de hemograma e fibrinogênio plasmático dentro dos valores de referência segundo Cowell e Tyler (1992).

Para o diagnóstico endoscópico da HPIE, foi utilizado um colonofibroscópio Olympus modelo CF 10L, acoplado a uma microcâmera Karl Storz modelo Endovision XL 20280130 NTSC e uma filmadora digital Sony modelo DCR HC-65. A endoscopia foi realizada de 30 a 90 minutos após o exercício.

Os animais foram divididos em dois grupos por meio de avaliação semi quantitativa para presença de sangue na traquéia, de acordo com Costa et al. (2004). Desta forma, o grupo 1 foi constituído por 26 animais com ausência de sangue na traquéia (sem HPIE, Grau 0), ao exame endoscópico e o Grupo 2 por 11 animais com HPIE caracterizada pela presença de sangue na traquéia em diferentes graus (1 a 3).

Para a obtenção do LT os animais foram colocados em brete e utilizando-se contenção física. O endoscópio, contendo a sonda para colheita transendoscópica (MILA® Delivery Catheter) em seu canal de trabalho, foi introduzido pelo meato nasal ventral em direção a traquéia. $A$ sonda foi posicionada na traquéia em porção distal, anterior a carina, de acordo com técnica utilizada por Whitwell e Greet (1984). Para a obtenção do LT, 20 a $40 \mathrm{~mL}$ de solução salina estéril foram instilados por meio de seringas plásticas estéreis (Injex®) e imediatamente aspirados. As amostras foram consideradas adequadas quando foi possível observar partículas suspensas ou filamentos de muco. O lavado obtido foi acondicionado em frascos cônicos graduados de $50 \mathrm{~mL}$, mantido sob refrigeração até o momento do processamento.

Alíquotas de $5 \mathrm{~mL}$ de lavado foram submetidas a centrifugação a $110 \mathrm{~g}$ por $5 \mathrm{~min}$. em centrífuga RDE® modelo MC-16. O sobrenadante foi dispensado e o sedimento de células utilizado para confecção das lâminas pela técnica de esfregaço linear, segundo Cowell e Tyler (1992). As lâminas fora fixadas em álcool metílico por 5 minutos e coradas pelo método de azul da Prússia.

Para a obtenção do ETH nas citologias de LT foi aplicada a metodologia descrita por Doucet e Viel (2002) para lavados broncoalveolares. Dessa forma, foram considerados com HPIE os animais cujo ETH foi igual ou maior que 75.

Foram obtidas as médias, os desvios-padrão e o coeficiente de variação para os valores de ETH segundo os Graus de HPIE encontrados à endosocopia.

\section{RESULTADOS E DISCUSSÃO}

Todos os 37 animais do estudo apresentaram presença de hemossiderófagos no LT. Esse fato reforça a necessidade da avaliação citológica semi quantitativa para a HPIE, uma vez que apenas a presença dessa célula não permite caracterizar a intensidade da enfermidade. De modo semelhante, Sweeney et al. (1992) obtiveram hemossiderófagos em lavados traqueais de $86 \%$ dos animais de corrida. McKane et al. (1993) detectaram essas células no lavado broncoalveolar de $90 \%$ dos animais 
examinados. Essa diferença provavelmente deve-se a colocação aplicada as lâminas, pois o azul da prússia utilizado em nosso estudo melhora a visualização dos hemossiderófagos. A elevada frequência com que os hemossiderófagos apareceram nos preparados citológicos já era esperada, pois segundo Whitwell e Greet (1984) todo equino submetido a exercício extenuante sofre de algum grau de hemorragia pulmonar.

Os resultados individuais para 0 escore de hemossiderina encontram-se na tabela 1. Considerando o valor de escore proposto por Doucet e Viel (2002), no grupo 1, a ocorrência de HPIE foi observada em $11,5 \%$ dos animais e no grupo 2 em 27,2\%.

Tabela 1- Valores individuais para o escore total de hemossiderina (ETH) na citologia de lavados traqueais dos equinos de pólo dos Grupos 1 $(n=26)$ e $2(n=11)$, após o exercício. Rio de Janeiro,

\begin{tabular}{|c|c|c|c|}
\hline \multicolumn{2}{|c|}{ GRUPO 1} & \multicolumn{2}{|c|}{ GRUPO 2} \\
\hline Animal & ETH & Animal & ETH \\
\hline 2 & 7 & $\overline{9}$ & 26,6 \\
\hline 4 & 16 & 21 & 32 \\
\hline 5 & 21 & 31 & 65,7 \\
\hline 6 & 31,1 & 34 & 16 \\
\hline 7 & 204,0 & 37 & 27 \\
\hline 8 & 21 & 3 & 17 \\
\hline 10 & 42,5 & 7 & 31 \\
\hline 11 & 23 & 13 & 40 \\
\hline 12 & 40 & 15 & 203,5 \\
\hline 13 & 79 & 19 & 84 \\
\hline 14 & 34 & 35 & 104 \\
\hline 16 & 59 & & \\
\hline 17 & 64 & & \\
\hline 18 & 16 & & \\
\hline 19 & 151 & & \\
\hline 21 & 6 & & \\
\hline 25 & 42 & & \\
\hline 26 & 42 & & \\
\hline 28 & 7 & & \\
\hline 29 & 28 & & \\
\hline 30 & 17 & & \\
\hline 31 & 27 & & \\
\hline 32 & 9 & & \\
\hline 33 & 11 & & \\
\hline 34 & 31 & & \\
\hline 36 & 7 & & \\
\hline
\end{tabular}

Os animais 7, 13 e 19 (Grupo 1) não apresentarem sangramento visível ao exame endoscópico pós jogo, porém apresentaram quadros citológicos de hemorragia pregressa importante, caracterizados por ETH maiores que 75 . É possível que a intensidade do exercício realizado neste dia não tenha sido intensa o suficiente a ponto de levar a sangramento perceptível. 0 tempo de permanência dos hemossiderófagos nas secreções após o episódio de sangramento ainda não foi completamente estabelecido em cavalos. Segundo Hinchcliff (2004), estas células estão presentes no LBA de 7 a 21 dias após o episódio de sangramento, informação também descrita por McKane e Slocombe (1999) em estudo com LBA e aspirado traqueal. Dessa forma é possível que estes animais tenham sofrido hemorragias prévias ao exame endoscópio ainda permanecendo o ETH elevado.

No animal 3 , o conjunto dos achados da endoscopia e citologia nos permite afirmar que trata-se de um animal que sofre episódios periódicos de hemorragia clinicamente relevante.

Doucet e Viel (2002) definiram como ponto de corte o valor para ETH acima de 75 a ser empregado em lavados broncoalveolares. Os mesmos autores afirmam ainda, que um escore acima de 150 parece ter uma especificidade de $100 \%$ na identificação da HPIE, mesmo em animais sem presença de sangue na endoscopia pósexercício. Da mesma forma, um ETH de 25 ou menos demonstrou sensibilidade de $100 \%$ em detectar os animais negativos. Os autores evidenciaram uma sensibilidade de $94 \%$ e especificidade de $88 \%$ para detecção da HPIE na citologia de lavado broncoalveolar.

Empregando o ponto de divisão estabelecido por Doucet e Viel, (2002), dos animais do Grupo 2 ( $n=11), 03$ 

hemorragia pulmonar induzida por exercício em cavalos de pólo

$(27,2 \%)$ tiveram ETH acima de 75 e 06 $(54,5 \%)$ tiveram abaixo de 75 e acima de 25 , sendo que somente 2 animais tiveram escore menor do que 25 . Os autores afirmam, ainda, que para propósitos de pesquisa outros pontos de corte mais específicos podem ser definidos, dependendo dos objetivos de triagem para seleção dos indivíduos do estudo.

Derksen et al. (1989) sugerem que a população celular nas vias aéreas pode diferir significativamente por causa da migração celular local para o lúmen aéreo. As vias aéreas de maior calibre podem transportar material originado tanto das vias aéreas condutoras como também da área de superfície alveolar. Logo, diferentes áreas contribuem com secreções de uma maneira não homogênea. Considerando também que - local onde comumente ocorrem episódios de hemorragia é o lobo caudal do pulmão, é provável que o LT seja um material mais diluído que o LBA quando consideramos a presença de hemossiderófagos. Portanto, é possível que em nosso estudo, a ocorrência da HPIE possa estar sendo subestimada pela utilização do ponto de corte empregado em LBA.

\section{CONCLUSÃO}

A utilização do escore total de hemossiderófagos pode ser considerado como uma importante ferramenta para diagnóstico da HPIE em equinos de pólo, podendo ser aplicada na rotina da clínica de equinos.

\section{REFERÊNCIAS}

BIAVA, J.S.;GONÇALVES, R.C.;BIONDO, A.W.; et al. Escore total de hemossiderófagos (THS) na hemorragia pulmonar induzida por exercício(EIPH) em cavalos quarto de milha.

Revista Acadêmica ciências agrárias ambientais, v.6, n.3, p.335-340, 2008.
COSTA, M.F.M.; THOMASSIAN,A.;

GOMES,T.S.;MAIA, E. Estudo da Hemorragia

Pulmonar induzida por esforço (HPIE) em cavalos de corrida PSI através da análise de 1889 endoscopias respiratórias após corrida. In:

Revista Brasileira de Ciência Veterinária, v.11, n.3, p.89-91.2004

COWELL RL.;TYLER RD, Diagnostic Cytology and Hematology of the Horse. 2nd edition. Mosby St Louis, 1992.

DeHEER, H.L.;McMANUS, P. Frequency and severity of tracheal hemosiderosis and association with underlying disease in 96 cats:2002-2003. Veterinary Clinical Pathology, v.34, n.1, p.17-22. 2005.

DERKSEN, F.J.; BROWN, C.M.; SONEA, I. Comparison of transtracheal aspirate and bronchoalveolar lavage cytology in 50 horses with chronic lung disease. Equine Veterinary Journal, v.21, n.1, p.23-26, 1989.

DOUCET, M.Y. \& VIEL, L. Clinical, radiographic, endoscopic, bronchoalveolar lavage and lung biopsy findings in horses with exercise-induced pulmonary hemorrhage.

Canadian Veterinary Journal, v.43, p.195-202, 2002

GOLDE, D.W.; DREW,W.L.; KLEIN,H.Z. Occult pulmonary hemorrhage in leukemia. British Medical Journal, v.2, p.166-168, 1975

HINCHCLIFF, K. W.; KANEPS, A. J.; GEOR, R. $J$. Equine sports medicine and surgery. Philadelphia: W. B. Saunders Company, 2004.

HODGSON, J.L; HODGSON, D.R. Tracheal Aspirates: Indications, Technique and Interpretation. In: ROBINSON, N.E. Current Therapy in Equine Medicine. Philadelphia: W. B. Saunders Company, 2003

HOFFMAN, A.M. Bronchoalveolar Lavage: Sampling Technique and Guidelines for Cytologic Preparation and Interpretation. Veterinary Clinics of North America: Equine Practice, v.24, n.2, p.423-435, 2008

MICHELOTTO JR, P. V.; BIAVA, J. S.; GONÇALVES, R. C; et al. Aspirado traqueal de cavalos clinicamente sadios da raça Quarto de Milha após prova de três tambores. Archives of Veterinary Science, v.12, n. 2, p.1-7, 2007.

MCGORUM, B.C.; DIXON, P.M.; RADOSTITS, O.M.; ABBOTT, J.A. Exame clínico do trato respiratório. In: RADOSTITS, O. M.; MAYHEW, 
I. G. J.; HOUSTON, D. M. (Ed.). Exame clínico e diagnóstico em veterinária. Rio de Janeiro: Guanabara Koogan. p. 231-269. 2002

MCKANE, S.A.; CANFIELD, P.J.; ROSE, R.J.

Equine bronchoalveolar lavage cytology: survey of thoroughbred racehorses in training.

Australian Veterinary Journal, v.70, n.11, p. 401-4,1993

MCKANE, S.A.; SLOCOMBE, R.F. Sequential changes in bronchoalveolar cytology after autologous blood inoculation. Equine

Veterinary Journal, Supplement, v.30, p 126130, 1999.

SWEENEY, C.R.; HUMBER, K.A. Cytologic findings of tracheobronchial aspirates from 66 thoroughbred racehorses. American Journal of Veterinary Research, v.53, n.7, p.1172-5,1992.

WHITWELL, K.E.; GREET, T.R.C. Collection and evaluation of tracheobronchial washes in the horse. Equine Veterinary Journal, v.16, n.6, p.499-508, 1984. 